\title{
SUSY $N$-SUPERGROUPS AND THEIR REAL FORMS
}

\author{
RITA FIORESI AND STEPHEN D. KWOK
}

\begin{abstract}
We study SUSY $N$-supergroups, $N=1$, 2 , their classification and explicit realization, together with their real forms. In the end, we give the supergroup of SUSY preserving automorphisms of $\mathbb{C}^{1 \mid 1}$ and we identify it with a subsupergroup of the SUSY preserving automorphisms of $\mathbb{P}^{1 \mid 1}$.
\end{abstract}

\section{INTRODUCTION}

SUSY curves represent a basic example in the theory of complex supermanifolds (see [1, 2, 19, 3, 22]). Since the seminal work by Manin [18, they have been studied in depth by several authors (see [7, 9, 20, 21, 17] and references therein). The presence of a SUSY structure on a complex supermanifold of dimension $1 \mid N$ makes the theory strikingly resemblant to the theory of ordinary Riemann surfaces and has sparkled questions on the corresponding moduli superspaces (see [9] and references therein) in connection with important theoretical physics applications (e.g. superstring perturbation theory [8, 23]).

In this paper we want to study the SUSY $N$-curves, which also admit a supergroup structure leaving invariant their SUSY structure, namely the SUSY $N$ supergroups. SUSY $N$-curves have been the object of study of several papers; most remarkably, in 20, 21 Rabin et al. study families of super elliptic curves over non trivial odd bases (see also Remark 2.6), focussing however on their complex structure only.

On the other hand, studying the real forms and the supergroup structure of SUSY curves gives the opportunity of exploiting representation theory for applications. The papers [5] and [6] carry out a thorough study of the real compact supergroups $S^{1 \mid 1}$ and $S^{1 \mid 2}$, called supercircles, in odd dimension 1 and 2, and their representation theory, together with the Peter-Weyl theorem. These supercircles are realized as real forms of $\left(\mathbb{C}^{1 \mid 1}\right)^{\times}$and $\left(\mathbb{C}^{1 \mid 2}\right)^{\times}$respectively, and in the case of $S^{1 \mid 1}$, we have a precise correspondence between the real structures and real forms of $\left(\mathbb{C}^{1 \mid 1}\right)^{\times}$and the SUSY preserving automorphisms of the SUSY 1-curve $\left(\mathbb{C}^{1 \mid 1}\right)^{\times}$. Since the study of one-parameter subgroups was the key for the construction of algebraic supergroups over arbitrary rings (see [10, 11, 12]), it is only reasonable

2010 Mathematics Subject Classification. 14M30, 17A70, 32C11, 58A50.

S. D. Kwok was partly supported by AFR grant No. 7718798 of the Luxembourg National Research Foundation. 
to expect that the understanding of SUSY $N$-supergroups may shed light on the super generalization of the Peter-Weyl theorem for arbitrary compact supergroups.

Our paper is organized as follows.

In Sections 2 and 3 we give the definition of SUSY $N$-supergroups and we classify them (Prop. 3.1). We also give an interpretation of the supergroup $\mathrm{SL}(1 \mid 1)$ as the SUSY 2-curve incidence supermanifold of the SUSY 1-curve $\left(\mathbb{C}^{1 \mid 1}\right)^{\times}$and its dual. In Section 4 we study real forms of SUSY $N$-supergroups of type 1 and classify them (Prop. 4.2 and Thm. 4.3). Finally in Section 5 we compute the supergroup of SUSY preserving automorphisms of $\mathbb{C}^{1 \mid 1}$ (Prop. 5.1.

Acknowledgments. We wish to thank professor P. Deligne for helpful comments. R. Fioresi, S. D. Kwok thank the University of Luxembourg and the University of Bologna for their wonderful hospitality while this work was done.

\section{The SUSY $N$-SUPERGRoups}

We want to study SUSY $N$-curves which also have a supergroup structure, which preserves the SUSY $N$-structure. In the following we shall use the notation and terminology as in [18, Ch. 2]. In particular, we understand a supermanifold $X$ as a locally ringed space, i.e., as a pair $\left(|X|, \mathcal{O}_{X}\right)$ consisting of a topological space and a sheaf of superrings.

$X$ is a $S U S Y 1$-curve if $X$ is a $1 \mid 1$ complex supermanifold and there is a $0 \mid 1$ distribution $\mathcal{D}$ such that the Frobenius map $\mathcal{D} \otimes \mathcal{D} \rightarrow T X / \mathcal{D}$ given by $Y_{1} \otimes Y_{2} \mapsto$ $\left[Y_{1}, Y_{2}\right](\bmod \mathcal{D})$ is an isomorphism. $X$ is a $S U S Y 2$-curve if it is a $1 \mid 2$ complex supermanifold and there are two $0 \mid 1$ distributions $\mathcal{D}_{i}$ such that $\left[\mathcal{D}_{i}, \mathcal{D}_{i}\right] \subset \mathcal{D}_{i}$ and the Frobenius map $\mathcal{D}_{1} \otimes \mathcal{D}_{2} \rightarrow T X /\left[\mathcal{D}_{1}, \mathcal{D}_{2}\right]$ is an isomorphism. In [18, Ch. 2], Manin provides local models for such distributions:

$$
\begin{aligned}
\mathcal{D} & =\zeta \partial_{z}+\partial_{\zeta} & & \text { on } X \text { a SUSY 1-curve } \\
\mathcal{D}_{1} & =\zeta_{2} \partial_{z}+\partial_{\zeta_{1}}, \quad \mathcal{D}_{2}=\zeta_{1} \partial_{z}+\partial_{\zeta_{2}} & & \text { on } X \text { a SUSY } 2 \text {-curve. }
\end{aligned}
$$

Definition 2.1. Let $X$ be a SUSY $N$-curve, with $0 \mid 1$ distribution(s) $\mathcal{D}_{i}$, where $i=1$ for $N=1$ and $i=1,2$ for $N=2$. We say that $X$ is a $S U S Y N$-supergroup if $X$ is a supergroup and the distribution(s) $\mathcal{D}_{i}$ are left invariant.

If $X$ and $Y$ are SUSY $N$-supergroups, we say that $f: X \longrightarrow Y$ is a morphism of SUSY $N$-supergroups if $f$ is a supergroup morphism and $f_{*}\left(\mathcal{D}_{i}\right)=\mathcal{D}_{j}$, that is, $f$ preserves the distributions or exchanges them.

We can immediately compute the Lie superalgebra of SUSY $N$-supergroups.

Proposition 2.2. Let $X$ be a SUSY N-supergroup. Then:

$$
\begin{aligned}
& \operatorname{Lie}(X)=\langle Z, C\rangle, \quad[Z, Z]=0, \quad \text { for } N=1, \\
& \operatorname{Lie}(X)=\left\langle Z_{1}, Z_{2}, C\right\rangle, \quad\left[Z_{1}, Z_{2}\right]=C, \quad \text { for } N=2,
\end{aligned}
$$

where $Z, Z_{i}$ are suitably chosen odd elements and all the brackets we do not write are assumed to be zero. 
Proof. For $N=1$ see [5]. Let $N=2, \mathcal{D}_{i}$ the left invariant distributions on the SUSY 2-supergroup $X$. Let $Z_{i} \in \operatorname{Lie}(X)$ be a left invariant (odd) generator of $\mathcal{D}_{i}$. We have $\operatorname{Lie}(X)=\left\langle Z_{1}, Z_{2},\left[Z_{1}, Z_{2}\right]\right\rangle$. Notice that in general $\left[\mathcal{D}_{i}, \mathcal{D}_{i}\right] \neq 0$; however, since $Z_{i} \in \operatorname{Lie}(X)$ and the bracket must preserve the parity, we have $\left[Z_{i}, Z_{i}\right]=0$. Let $C:=\left[Z_{1}, Z_{2}\right]$. The Jacobi identity gives immediately that $C$ is central.

Since SUSY $N$-supergroups are in particular supergroups, we shall use freely the formalism of super Harish-Chandra pairs (SHCP); see [3, Ch. 7] and [4, 15] for more details.

Proposition 2.3. Let $X, X^{\prime}$ be $S U S Y N$-supergroups and $\widetilde{X}, \widetilde{X}^{\prime}$ their underlying reduced groups. Then $X \cong X^{\prime}$ if and only if $\widetilde{X} \cong \widetilde{X}^{\prime}$.

Proof. Suppose $f: X \rightarrow X^{\prime}$ is an isomorphism. Then $\tilde{X}$ and $\tilde{X}^{\prime}$ are isomorphic.

Conversely, suppose $|f|: \widetilde{X} \rightarrow \widetilde{X}^{\prime}$ is an isomorphism. $|f|: \widetilde{X} \rightarrow \widetilde{X}^{\prime}$ lifts to the universal covers, giving an isomorphism $\tilde{f}: \mathbb{C} \rightarrow \mathbb{C}$ which fixes the origin. By a standard result from one-variable complex analysis, $\tilde{f}$ is multiplication by a nonzero scalar $\lambda$. If $N=1$, define the super Lie algebra morphism $\varphi: \mathfrak{g} \rightarrow \mathfrak{g}$ by $C \mapsto \lambda C^{\prime}, Z \mapsto \sqrt{\lambda} Z^{\prime}$. Clearly $\left.\varphi\right|_{\mathfrak{g}_{0}}=d|f|$, so $F:=(|f|, \varphi)$ is an isomorphism of SHCP, and hence $X \cong X^{\prime}$ as supergroups. By construction, $d F\left(\mathcal{D}_{e}\right)=\mathcal{D}_{e}^{\prime}$ at the identity $e$. However, as $\mathcal{D}, \mathcal{D}^{\prime}$ are left-invariant and $F$ is a supergroup isomorphism, we have $d F\left(\mathcal{D}_{p}\right)=\mathcal{D}_{p}^{\prime}$ at every point $p$ of $X$, hence $F$ is an isomorphism of SUSY $N$-supergroups.

If $N=2$, define the super Lie algebra morphism $\varphi: \mathfrak{g} \rightarrow \mathfrak{g}$ by $C \mapsto \lambda C^{\prime}$, $Z_{1} \mapsto \sqrt{\lambda} Z_{1}^{\prime}, Z_{2} \mapsto \sqrt{\lambda} Z_{2}^{\prime}$. Again, $\left.\varphi\right|_{\mathfrak{g}_{0}}=d|f|$, so $F:=(|f|, \varphi)$ is an isomorphism of SHCP. This shows that $X \cong X^{\prime}$ as supergroups; however, reasoning as above, we obtain our result.

Corollary 2.4. Let $X$ be a SUSY $N$-supergroup. Then $X=\mathbb{C}^{1 \mid N}$ or $X \cong \mathbb{C}^{1 \mid N} / G$, where $G$ is either a rank 1 free abelian subgroup of $\mathbb{C}^{1 \mid N}$ or a lattice in $\mathbb{C}^{1 \mid N}$. Furthermore, $X$ inherits its SUSY N-structure from its universal cover $\mathbb{C}^{1 \mid N}$.

Proof. If $X$ is simply connected, then $X=\mathbb{C}^{1 \mid N}$. Assume $X$ is not simply connected. The universal cover of $X$ is readily seen to be $\mathbb{C}^{1 \mid N}$. The kernel of the covering morphism $\pi: \mathbb{C}^{1 \mid N} \rightarrow X$ is a $0 \mid 0$-subsupergroup $G$ of $\mathbb{C}^{1 \mid N}$ and, by a classical argument, is either a rank 1 free abelian subgroup of $\mathbb{C}^{1 \mid N}$ or a lattice in $\mathbb{C}^{1 \mid N}$. So $X \cong \mathbb{C}^{1 \mid N} / G$.

Definition 2.5. We say that $X$ is a SUSY $N$-supergroup of type 1 (resp. type 2) if $X=\mathbb{C}^{1 \mid N} / G$, where $G$ is a rank 1 free abelian subgroup (resp. a lattice) in $\mathbb{C}^{1 \mid N}$.

We focus now on the type 1 SUSY $N$-supergroups, leaving the discussion of type 2, which is more involved, for a future development. In fact, such supergroups, as supermanifolds (unlike the 1|1 case), are not split, so they are technically more difficult to treat and require new methods (in the $1 \mid 1$ case we can just view the odd coordinate as a line bundle over the reduced manifold).

We end this section with a remark relating our treatment with [20, 21]. 
Remark 2.6. In 20, 21, Rabin et al. consider families $X \rightarrow \mathcal{B}$ of SUSY 1-elliptic curves over a base superspace $\mathcal{B}=(\{p t\}, \Lambda)$, where $\Lambda$ is a non trivial Grassmann algebra. In this setting, families of SUSY 1-curves do not admit any natural structure of supergroup over $\mathcal{B}$.

This is consistent with our treatment, because we work in the absolute setting, i.e., taking $\mathcal{B}=(\{p t\}, \mathbb{C})$, so it is possible to endow a SUSY 1-elliptic curve with the supergroup structure inherited from its universal cover $\mathbb{C}^{1 \mid 1}$ (Cor. 2.4).

\section{SUSY $N$-SUPERGROUPS OF TYPE 1}

We want to classify the SUSY $N$-supergroups of type 1 and relate them to Manin's approach to SUSY curves. We shall use interchangeably the formalisms of functor of points and also of super Harish-Chandra pairs (SHCP).

Proposition 3.1. Up to isomorphism, for $N=1,2$ fixed, we have only two SUSY $N$-supergroups of type 1.

(1) For $N=1$ they are $\left(\mathbb{C}^{1 \mid 1}\right)^{\times}$and $\mathbb{C}^{1 \mid 1}$ with group law respectively:

$$
\begin{aligned}
(w, \eta) \cdot\left(w, \eta^{\prime}\right) & =\left(w w^{\prime}+\eta \eta^{\prime}, w \eta^{\prime}+\eta w^{\prime}\right), \\
(z, \zeta) \cdot\left(z^{\prime}, \zeta^{\prime}\right) & =\left(z+z^{\prime}+\zeta \zeta^{\prime}, \zeta+\zeta^{\prime}\right) .
\end{aligned}
$$

(2) For $N=2$ they are $\left(\mathbb{C}^{1 \mid 2}\right)^{\times}$and $\mathbb{C}^{1 \mid 2}$ with group laws:

$$
\begin{aligned}
(v, \xi, \eta) \cdot\left(v^{\prime}, \xi^{\prime}, \eta^{\prime}\right) & =\left(v v^{\prime}+\eta \xi^{\prime}, v \xi^{\prime}+\xi v^{\prime}+\xi v^{-1} \eta \xi^{\prime}, \eta v^{\prime}+v \eta^{\prime}+\eta \xi^{\prime} v^{\prime-1} \eta^{\prime}\right), \\
(z, \zeta, \chi) \cdot\left(z^{\prime}, \zeta^{\prime}, \chi^{\prime}\right) & =\left(z+z^{\prime}+\zeta \chi^{\prime}, \zeta+\zeta^{\prime}, \chi+\chi^{\prime}\right) .
\end{aligned}
$$

Proof. For $N=1$ the statements are contained in [5], provided that one verifies left invariance, which is a straightforward check. Let $N=2, \mathcal{D}_{i}$ the left invariant distributions on the SUSY 2-supergroup $X$. Let $D_{i} \in \operatorname{Lie}(X)$ be a left invariant (odd) generator of $\mathcal{D}_{i}$. By 2.2 we have $\operatorname{Lie}(X)=\left\langle D_{1}, D_{2},\left[D_{1}, D_{2}\right]\right\rangle$. The given group laws correspond to the Lie superalgebra we have obtained respectively for $G_{0}=\mathbb{C}^{\times}$and $G_{0}=\mathbb{C}$. For example, let us compute the Lie superalgebra structure for $\left(\mathbb{C}^{1 \mid 2}\right)^{\times}$(the case of $\mathbb{C}^{1 \mid 2}$ is similar). The tangent space is $\mathbb{C}^{1 \mid 2}$ and let $e_{1}, \mathcal{E}_{1}, \mathcal{E}_{2}$ be the canonical basis. The corresponding left invariant vector fields in the global coordinates $(v, \xi, \eta)$ are:

$$
\begin{aligned}
D_{1} & =\left(d \ell_{(u, \mu, \nu)}\right)_{(1,0,0)} \mathcal{E}_{1}=v \partial_{\eta} \\
D_{2} & =\left(d \ell_{(u, \mu, \nu)}\right)_{(1,0,0)} \mathcal{E}_{2}=-\eta \partial_{v}+\left(v+\xi v^{-1} \eta\right) \partial_{\xi} \\
E & =\left(d \ell_{(u, \mu, \nu)}\right)_{(1,0,0)} e_{1}=v \partial_{v}+\xi \partial_{\xi}+\eta \partial_{\eta} .
\end{aligned}
$$

As one can readily check, $\left[D_{1}, D_{2}\right]=-2 E$ (so we set $C=-2 E$ ) and $\left[D_{i}, D_{i}\right]=0$.

Remark 3.2. We can interpret the multiplicative and additive SUSY 2-supergroups using matrix supergroups. $\left(\mathbb{C}^{1 \mid 2}\right)^{\times}$is $\mathrm{SL}(1 \mid 1)$. In functor of points notation:

$$
\left(\mathbb{C}^{1 \mid 2}\right)^{\times}(T)=\mathrm{SL}(1 \mid 1)(T)=\left\{\left(\begin{array}{ll}
u & \xi \\
\eta & v
\end{array}\right) \mid v^{-1}\left(u-\xi v^{-1} \eta\right)=1\right\} .
$$


$\mathbb{C}^{1 \mid 2}$ is the subgroup of $\operatorname{SL}(2 \mid 1)$ given in the functor of points notation by

$$
\mathbb{C}^{1 \mid 2}(T)=\left\{\left(\begin{array}{lll}
1 & z & \zeta \\
0 & 1 & 0 \\
0 & \chi & 1
\end{array}\right)\right\}
$$

For example, let us check the second of these statements, the first one being the same. We can verify the claim reasoning in terms of the functor of points. So we have to compute:

$$
\left(\begin{array}{lll}
1 & z & \zeta \\
0 & 1 & 0 \\
0 & \chi & 1
\end{array}\right) \cdot\left(\begin{array}{ccc}
1 & z^{\prime} & \zeta^{\prime} \\
0 & 1 & 0 \\
0 & \chi^{\prime} & 1
\end{array}\right)=\left(\begin{array}{ccc}
1 & z^{\prime}+z+\zeta \chi^{\prime} & \zeta+\zeta^{\prime} \\
0 & 1 & 0 \\
0 & \chi+\chi^{\prime} & 1
\end{array}\right)
$$

which is precisely the multiplication as in 3.1. This approach can be helpful in calculations. We leave to the reader the straightforward checks regarding the group law of the first statement.

We now want to interpret some of the discussion in [18, Sec. 6] in the framework of SUSY supergroups. Let $X$ be a SUSY 1-curve and $\widehat{X}$ its dual. The $T$-points of $\widehat{X}$ are the $0 \mid 1$ subvarieties of $X(T)$. Let $\Delta$ be the superdiagonal subscheme of $X \times \widehat{X}$. It is locally defined by the incidence relation

$$
z-z^{\prime}-\zeta^{\prime} \zeta=0
$$

where $(z, \zeta)$ and $\left(z^{\prime}, \zeta^{\prime}\right)$ are local coordinates of $X$ and $\widehat{X}$, respectively (see [18. Def. 6.2]). $\Delta$ is a SUSY 2-curve, with distributions $\mathcal{D}_{1}, \mathcal{D}_{2}$, and we have the commutative diagram

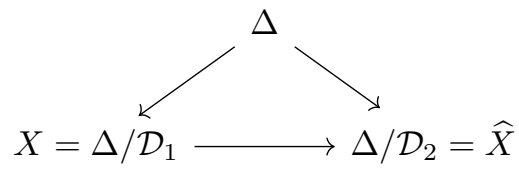

where $\Delta / \mathcal{D}_{i}$ means the superspace whose reduced space is $|\Delta|$, and whose structure sheaf is the subsheaf of $\mathcal{O}_{\Delta}$ consisting of sections which are invariant under $\mathcal{D}_{i}$. Specifying a SUSY-1 structure on $X$ gives an isomorphism $X \cong \widehat{X}$.

On $\left(\mathbb{C}^{1 \mid 2}\right)^{\times}$we have the global SUSY 2-structure:

$$
D_{1}=\partial_{\zeta_{1}}+\zeta_{2} \partial_{z}, \quad D_{2}=\partial_{\zeta_{2}}+\zeta_{1} \partial_{z} .
$$

Clearly $D_{1}^{2}=D_{2}^{2}=0$ and $\left[D_{1}, D_{2}\right]=2 \partial_{z}$. In Remark 3.2 we have viewed $\left(\mathbb{C}^{1 \mid 2}\right)^{\times}$ as the supergroup $\mathrm{SL}(1 \mid 1)$. The condition on the Berezinian is the (global) incidence relation $(3.2)$ and allows us to identify $\operatorname{SL}(1 \mid 1)$ with $\Delta$ for $X=\left(\mathbb{C}^{1 \mid 1}\right)^{\times}$and GL(1|1) with $X \times \widehat{X}$. Notice that in our special case, namely for $X=\widehat{X}=\left(\mathbb{C}^{1 \mid 1}\right)^{\times}$, we have that $X, \widehat{X} \subset \mathrm{SL}(1 \mid 1)$ as the subsupergroups

$$
X(T)=\left\{\left(\begin{array}{ll}
x & \xi \\
\xi & x
\end{array}\right)\right\}, \quad \widehat{X}(T)=\left\{\left(\begin{array}{cc}
y & \eta \\
-\eta & y
\end{array}\right)\right\} .
$$


These inclusions correspond to the Lie superalgebra inclusions

$$
\langle C, U=E+F\rangle, \quad\langle C, V=E-F\rangle \subset\langle C, E, F\rangle=\operatorname{sl}(1 \mid 1),
$$

where $C, E, F$ are the usual generators for $\operatorname{sl}(1 \mid 1)$, namely

$$
[C, E]=[C, F]=[E, E]=[F, F]=0, \quad[E, F]=C .
$$

\section{REAL FORMS OF SUSY SUPERGROUPS}

We want to study the real forms of SUSY $N$-supergroups of type 1 . In [5] and [6] we proved that, up to isomorphism, there is one real form of the SUSY 1-supergroup $\left(\mathbb{C}^{1 \mid 1}\right)^{\times}$and the corresponding involution is the composition of complex conjugation and the SUSY preserving automorphisms $P_{ \pm}$. We wish to prove a similar result for the SUSY 2-supergroups.

Definition 4.1. Let $X$ be a SUSY 2-curve with distributions $\mathcal{D}_{i}$. We say that an automorphism $\phi: X \longrightarrow X$ is $S U S Y$ preserving if $\phi_{*}\left(\mathcal{D}_{i}\right)=\mathcal{D}_{j}$, that is, if $\phi$ preserves individually each distribution or exchanges them. If $X$ is a SUSY 2-supergroup, we additionally require $\phi$ to be a supergroup automorphism.

Notice that in a SUSY 2-curve the roles of $\mathcal{D}_{1}$ and $\mathcal{D}_{2}$ are interchangeable; this forces us to give such a definition of SUSY preserving automorphisms.

We start our discussion by observing that, up to isomorphism, there is only one real form of the Lie superalgebra $\operatorname{sl}(1 \mid 1)$ with compact even part. In fact, assume $\mathfrak{g}_{\mathbb{R}}=\operatorname{span}_{\mathbb{R}}\{i C, U, V\}$ is such real form, with central even element $i C$ (see $(3.3)$ ). If $U=a E+b F, V=c E+d F$, there is no loss of generality in assuming $a=1$ because $E \mapsto a^{-1} E, F \mapsto a F, C \mapsto C$ is a Lie superalgebra automorphism of $\operatorname{sl}(1 \mid 1)$. Assume further $[U, U] \neq 0$ (when both $[U, U]=[V, V]=0$ we leave to the reader the easy check of what the real form is). Then we have that $b=i$, up to a constant, that we incorporate in $C$. An easy calculation shows that $V=i E-F$, hence we have proven the following proposition.

Proposition 4.2. Up to isomorphism, there is a unique real form of $\mathrm{sl}(1 \mid 1)$ with compact even part, namely

$$
\operatorname{su}(1 \mid 1)=\operatorname{span}_{\mathbb{R}}\{i C, U=E+i F, V=i E-F\} \subset \operatorname{sl}(1 \mid 1) .
$$

We can then state the theorem giving all real forms of SUSY 2-supergroups with compact support.

Theorem 4.3. Up to isomorphism, there exists a unique real form of the SUSY 2 -supergroup $\mathrm{SL}(1 \mid 1)$ and it is obtained with an involution $\sigma=c \circ \phi$, where $\phi$ is a SUSY preserving automorphism and $c$ is a complex conjugation. Explicitly,

$$
\sigma\left(\begin{array}{ll}
a & \beta \\
\gamma & d
\end{array}\right)=\left(\begin{array}{cc}
\bar{d}^{-1} & -i \bar{a}^{-2} \bar{\gamma} \\
-i \bar{a}^{-2} \bar{\beta} & \bar{a}^{-1}
\end{array}\right) .
$$

Proof. We first check that the given $\sigma$ is of the prescribed type, namely that

$$
\phi\left(\begin{array}{ll}
a & \beta \\
\gamma & d
\end{array}\right)=\left(\begin{array}{cc}
d^{-1} & -i a^{-2} \gamma \\
-i a^{-2} \beta & a^{-1}
\end{array}\right)
$$


is a SUSY preserving automorphism. The fact that $\phi$ is a supergroup automorphism is a simple check, that can be verified using the functor of point formalism, namely one sees that

$$
\phi\left(\left(\begin{array}{ll}
a & \beta \\
\gamma & d
\end{array}\right) \cdot\left(\begin{array}{ll}
a^{\prime} & \beta^{\prime} \\
\gamma^{\prime} & d^{\prime}
\end{array}\right)\right)=\left(\begin{array}{cc}
d^{-1} & -i a^{-2} \gamma \\
-i a^{-2} \beta & a^{-1}
\end{array}\right)\left(\begin{array}{cc}
d^{\prime-1} & -i a^{\prime-2} \gamma \\
-i a^{\prime-2} \beta & a^{\prime-1}
\end{array}\right) .
$$

Since $\phi$ is a supergroup morphism, $d \phi$ preserves left invariant vector fields (see [3. Ch. 7]), hence it preserves the SUSY structure.

As for uniqueness, by Prop. 4.2 we know there exists a unique real form su(1|1) of $\operatorname{sl}(1 \mid 1)=\operatorname{Lie}(\mathrm{SL}(1 \mid 1))$ and one readily checks $\operatorname{su}(1 \mid 1)=\operatorname{Lie}(\mathrm{SU}(1 \mid 1))$. By the equivalence of categories in SHCP theory we obtain the result.

\section{The SUSY PRESERVING AUTOMORPhisms OF $\mathbb{C}^{1 \mid 1}$}

Let our notation and terminology be as in [5, 6].

On $\mathbb{C}^{1 \mid 1}$ we have the globally defined SUSY structure given by the vector field

$$
D=\partial_{\zeta}+\zeta \partial_{z},
$$

where $(z, \zeta)$ are the global coordinates. This structure is unique up to isomorphism (see [13, Sec. 4]). We want to determine the supergroup of automorphism of $\mathbb{C}^{1 \mid 1}$ preserving such SUSY structure. We will denote it with $\operatorname{Aut}_{\text {SUSY }}\left(\mathbb{C}^{1 \mid 1}\right)$. In the work [13] we have provided the $\mathbb{C}$-points of such supergroup; they are obtained by looking at the transformations leaving invariant the 1-form

$$
s=d z-\zeta d \zeta
$$

and are given by the endomorphisms

$$
F(z, \zeta)=(a z+b, \sqrt{a} \zeta)
$$

We can identify the $\mathbb{C}$-points of the supergroup of SUSY preserving automorphisms with the matrix group

$$
\operatorname{Aut}_{\mathrm{SUSY}}\left(\mathbb{C}^{1 \mid 1}\right)(\mathbb{C})=\left\{\left(\begin{array}{ccc}
c & d & 0 \\
0 & c^{-1} & 0 \\
0 & 0 & 1
\end{array}\right) \mid a, b \in \mathbb{C}\right\} \subset \operatorname{Aut}_{\mathrm{SUSY}}\left(\mathbb{P}^{1 \mid 1}\right)(\mathbb{C}) .
$$

This is a subgroup of the $\mathbb{C}$-points of the SUSY preserving automorphisms of the SUSY 1-curve $\mathbb{P}^{1 \mid 1}$, namely those fixing the point at infinity (see [13, Sec. 5]). In such identification $a=c^{2}$ and $b=d c$. Notice that, though $\operatorname{Aut}_{\mathrm{SUSY}}\left(\mathbb{C}^{1 \mid 1}\right)(\mathbb{C})$ is a matrix group, it is not obvious that also $\operatorname{Aut}_{\mathrm{SUSY}}\left(\mathbb{C}^{1 \mid 1}\right)$ should be, since we are looking at the SUSY preserving automorphisms of $\mathbb{C}^{1 \mid 1}$ as supermanifold morphisms. Nevertheless we will show that this is the case.

An automorphism $F: \mathbb{C}^{1 \mid 1} \longrightarrow \mathbb{C}^{1 \mid 1}$ induces an automorphism $F^{*}: \mathcal{O}\left(\mathbb{C}^{1 \mid 1}\right) \longrightarrow$ $\mathcal{O}\left(\mathbb{C}^{1 \mid 1}\right)$ of the superalgebra of global sections. $F$ is SUSY preserving if and only if

$$
F^{*} \circ D=k D \circ F^{*},
$$

where $D$ is now interpreted as a derivation of $\mathcal{O}\left(\mathbb{C}^{1 \mid 1}\right)$ and $k$ is a suitable constant. We first consider the infinitesimal picture and compute $\operatorname{Lie}\left(\operatorname{Aut}_{\mathrm{SUSY}}\left(\mathbb{C}^{1 \mid 1}\right)\right)$. By 
(5.1), Lie $\left(\operatorname{Aut}_{\mathrm{SUSY}}\left(\mathbb{C}^{1 \mid 1}\right)\right)_{0}$ is 2 dimensional, and as one can readily check, it is spanned by the two even vector fields

$$
U_{1}=2 z \partial_{z}+\zeta \partial_{\zeta}, \quad U_{2}=\partial_{z} .
$$

We hence only need to compute Lie $\left(\operatorname{Aut}_{\mathrm{SUSY}}\left(\mathbb{C}^{1 \mid 1}\right)\right)_{1}$.

Proposition 5.1. Lie(Aut $\left.{ }_{\mathrm{SUSY}}\left(\mathbb{C}^{1 \mid 1}\right)\right)$ is the Lie subsuperalgebra of the vector fields on $\mathbb{C}^{1 \mid 1}$ spanned by

$$
U_{1}=2 z \partial_{z}+\zeta \partial_{\zeta}, \quad U_{2}=\partial_{z}, \quad V=\zeta \partial_{z}-\partial_{\zeta}
$$

with brackets

$$
[V, V]=2 U_{2}, \quad\left[U_{2}, U_{1}\right]=-2 U_{1}, \quad\left[U_{2}, V\right]=-V, \quad\left[U_{1}, V\right]=0 .
$$

Proof. Consider $I+\theta \chi$, for $\chi \in \operatorname{Lie}\left(\operatorname{Aut}_{\mathrm{SUSY}}\left(\mathbb{C}^{1 \mid 1}\right)\right)_{1}$. The condition $5.2 \mathrm{im}$ mediately gives that the odd derivation $\chi^{*}$ of $\mathcal{O}\left(\mathbb{C}^{1 \mid 1}\right)$ induced by $\chi$ must satisfy $\left[\chi^{*}, D\right]=0$. A small calculation gives then the result.

In the super Harish-Chandra pair (SHCP) formalism, we can immediately write the supergroup of SUSY preserving automorphism:

$$
\operatorname{Aut}_{\mathrm{SUSY}}\left(\mathbb{C}^{1 \mid 1}\right)=\left(\operatorname{Aut}_{\mathrm{SUSY}}\left(\mathbb{C}^{1 \mid 1}\right)(\mathbb{C}), \operatorname{Lie}\left(\operatorname{Aut}_{\mathrm{SUSY}}\left(\mathbb{C}^{1 \mid 1}\right)\right)\right.
$$

The next proposition identifies such supergroup with a natural subsupergroup of $\operatorname{Aut}_{\mathrm{SUSY}}\left(\mathbb{P}^{1 \mid 1}\right)=\operatorname{SpO}(2 \mid 1)$ (see [14]) using the more geometric functor of points approach.

Proposition 5.2. Aut $\mathrm{SuSY}_{\mathrm{SU}}\left(\mathbb{C}^{1 \mid 1}\right)$ is the stabilizer subsupergroup in $\mathrm{SpO}(2 \mid 1)$ of the point at infinity:

$$
\operatorname{Aut}_{\mathrm{SUSY}}\left(\mathbb{C}^{1 \mid 1}\right)(T)=\left\{\left(\begin{array}{ccc}
c & d & \gamma \\
0 & c^{-1} & 0 \\
0 & c^{-1} \gamma & 1
\end{array}\right) \mid c, d \in \mathcal{O}(T)_{0}, \gamma \in \mathcal{O}(T)_{1}\right\}
$$

$T \in(\text { smflds })_{\mathbb{R}}$.

Proof. The first statement is an immediate consequence of Proposition 5.1. As for the second one, consider the subgroup $G$ of $\operatorname{SpO}(2 \mid 1)=\operatorname{Aut}_{\mathrm{SUSY}}\left(\mathbb{P}^{1 \mid 1}\right)$ that fixes the point at infinity. Its functor of points is given by

$$
G(T)=\left\{\left(\begin{array}{ccc}
c & d & \gamma \\
0 & c^{-1} & 0 \\
0 & c^{-1} \gamma & 1
\end{array}\right) \mid c, d \in \mathcal{O}(T)_{0}, \gamma \in \mathcal{O}(T)_{1}\right\}
$$

$G$ is representable and its $\operatorname{SHCP}$ coincides with $\operatorname{Aut}_{\mathrm{SUSY}}\left(\mathbb{C}^{1 \mid 1}\right)$, because $\operatorname{Lie}(G)$ $=\operatorname{Lie}\left(\operatorname{Aut}_{\mathrm{SUSY}}\left(\mathbb{C}^{1 \mid 1}\right),|G|=\operatorname{Aut}_{\mathrm{SUSY}}\left(\mathbb{C}^{1 \mid 1}\right)(\mathbb{C})\right.$, and we have the compatibility conditions. 


\section{REFERENCES}

[1] F. A. Berezin, Introduction to Superanalysis. D. Reidel, Dordrecht, 1987. MR 0914369

[2] F. A. Berezin, D. A. Leĭtes, Supermanifolds, Dokl. Akad. Nauk SSSR 224 (1975), no. 3, 505-508. MR 0402795

[3] C. Carmeli, L. Caston, R. Fioresi, Mathematical Foundations of Supersymmetry, EMS Series of Lectures in Mathematics. European Mathematical Society (EMS), Zürich, 2011. MR 2840967

[4] C. Carmeli, R. Fioresi, Superdistributions, analytic and algebraic super Harish-Chandra pairs. Pacific J. Math. 263 (2013), no. 1, 29-51. MR 3069075

[5] C. Carmeli, R. Fioresi, S. D. Kwok, SUSY structures, representations and Peter-Weyl theorem for $S^{1 \mid 1}$, J. Geom. Phys. 95 (2015), 144-158. MR 3357828

[6] C. Carmeli, R. Fioresi, S. D. Kwok, The Peter-Weyl theorem for SU(1|1). p-Adic Numbers Ultrametric Anal. Appl. 7 (2015), no. 4, 266-275. MR 3418794.

[7] P. Deligne, J. W. Morgan, Notes on supersymmetry (following Joseph Bernstein), in: Quantum Fields and Strings: A Course for Mathematicians, 41-97, Amer. Math. Soc., Providence RI, 1999. MR 1701597

[8] E. D'Hoker, D. H. Phong, The geometry of string perturbation theory, Rev. Modern Phys. 60 (1988), no. 4, 917-1065. MR 0969998

[9] R. Donagi, E. Witten, Supermoduli space is not projected, in: String-Math 2012, 19-71, Proc. Sympos. Pure Math., 90, Amer. Math. Soc., Providence RI, 2015. MR 3409787

[10] R. Fioresi, F. Gavarini, Algebraic supergroups with Lie superalgebras of classical type. J. Lie Theory 23 (2013), no. 1, 143-158. MR 3060770.

[11] R. Fioresi, F. Gavarini, Chevalley supergroups. Mem. Amer. Math. Soc. 215 (2012), no. 1014. MR 2918543

[12] R. Fioresi, F. Gavarini, On the construction of Chevalley supergroups, in: Supersymmetry in Mathematics and Physics, 101-123, Lecture Notes in Math., 2027, Springer, Heidelberg, 2011. MR 2906339

[13] R. Fioresi, S. D. Kwok, On SUSY curves, in: Advances in Lie Superalgebras, 101-119, Springer INdAM Series, 7, Springer, Cham, 2014. MR 3205084.

[14] R. Fioresi, S. D. Kwok, The projective linear supergroup and the SUSY preserving automorphisms of $\mathbb{P}^{1 \mid 1}$, Pacific J. Math. 295 (2018), no. 2, 385-401. MR 3788793.

[15] F. Gavarini, Lie supergroups vs. super Harish-Chandra pairs: a new equivalence, preprint, 2016. arXiv:1609.02844 [math.RA].

[16] D. A. Leŭtes, Introduction to the theory of supermanifolds, Russian Math. Surveys 35 (1980), no. 1, 1-64. MR 0565567

[17] A. M. Levin, Supersymmetric elliptic curves, Funct. Anal. Appl. 21 (1987), no. 3, $243-244$. MR 0911782

[18] Y. I. Manin, Topics in Non-commutative Geometry. Princeton University Press, 1991. MR 1095783

[19] Y. I. Manin, Gauge Field Theory and Complex Geometry. Springer-Verlag, Berlin, 1988. MR 0954833

[20] J. M. Rabin, P. G. O. Freund, Supertori are algebraic curves, Comm. Math. Phys. 114 (1988), no. 1, 131-145. MR 0925525

[21] J. M. Rabin, Super elliptic curves, J. Geom. Phys. 15 (1995), no. 3, 252-280. MR 1316333 
[22] V. S. Varadarajan, Supersymmetry for Mathematicians: An Introduction, Courant Lecture Notes in Mathematics, 11. New York University, Courant Institute of Mathematical Sciences, New York; American Mathematical Society, Providence, RI, 2004. MR 2069561

[23] E. Witten, Superstring perturbation theory revisited, preprint, 2012. arXiv:1209.5461 [hepth].

\section{R. Fioresi ${ }^{凶}$}

Dipartimento di Matematica, Università di Bologna, Piazza di Porta S. Donato, 5. 40126

Bologna, Italy

rita.fioresi@UniBo.it

S. D. Kwok

Department of Biostatistics, UCLA Fielding School of Public Health, 650 Charles E. Young Dr. South, 51-254 CHS, Los Angeles, CA 90095-1772

sdkwok2@gmail.com

Received: June 15, 2018

Accepted: January 2, 2019 